\title{
OPTIMIZATION OF GEOMETRICAL PARAMETERS AND ESTIMATION OF KINEMATIC ACCURACY OF RESOURCE EFFECTIVE PLANETARY TRANSMISSION
}

\author{
Alexandr V. Kapitonov \\ Belarusian-Russian University, \\ Mogilev, Republic of Belarus \\ kavbru@gmail.com
}

\begin{abstract}
The purpose of the research is to study how the shape of a geometric profile of the periodic raceway affects kinematic accuracy and smooth operation of the resourceeffective planetary radial plunger transmission with intermediate rolling bodies. The shape and computer model for a geometric profile of the periodic raceway of the planetary radial plunger transmission are proposed. The profile under study is constructed from the equation of a periodic curve in the form of a circle with an offset center. The transmission with the proposed profile is characterized by increased kinematic accuracy and smoothness of operation. A comparative analysis of kinematic errors of the planetary radial plunger transmission was performed for two profiles of raceways having different geometric shapes, and diagrams were constructed. Kinematic parameters of the transmission with the periodic raceway profile in the form of an eccentrically offset circle were improved. It was established that the use of the planetary radial plunger transmission with the periodic raceway profile constructed from the equation of a periodic curve in the form of a circle with an offset center significantly improves (three times or more) such transmission characteristics as kinematic accuracy and operation smoothness compared to the geometric profile in the form of arcs of circles.
\end{abstract}

Keywords - planetary transmission, periodic raceway, kinematic accuracy

\section{INTRODUCTION}

Planetary cycloid transmissions are the most promising mechanisms that are used in various automated systems, mechatronic devices and industrial robots. They differ in their design and include the group of resource-effective planetary radial plunger transmissions with intermediate rolling bodies in the form of satellite balls having small mass-dimensional characteristics, large gear ratios, small axial dimensions, shaft coincidence and other advantages.

The history of their research and the classification of these transmissions are presented in a number of research papers [15]. The studies of cycloid transmissions were conducted by scientists from different countries and are still continuing. Dynamic models of cycloid speed reducers of new designs with improved performance parameters were developed [6-9].
The study of kinematic accuracy and the estimation of the effect of manufacturing errors on the load-carrying capacity of transmissions were performed [10-12]. In order to improve their kinematic and dynamic characteristics, new designs and computer models were developed based on the analysis of patents and literature in the subject area [13-15].

\section{RESEARCH RESULTS AND THEIR DISCUSSION}

A raceway of the planetary radial plunger transmission, along which satellites move, can be made in the form of sinusoids, cycloids, offset circles and other curves closed in the plane. However, the most technological design is a periodic raceway in the form of an offset circle, because a bearing or a ring can be used as an input member instead of a cam of complex geometric shape.

In these transmissions, the raceways are designed in the form of arcs of circles to further improve their manufacturability. This simplifies their manufacture, but reduces kinematic accuracy and smoothness of operation, and impairs contact of parts in the engagement.

In order to improve the kinematic characteristics and high manufacturability of the transmissions under investigation, we propose to construct the geometry of the periodic raceway profile, along which satellites move, based on the equation of a periodic curve in the form of a circle with an offset center:

$$
\rho_{2}=\sqrt{R^{2}-A^{2} \sin ^{2} z_{2} \varphi}+A \cos z_{2} \varphi,
$$

where $\rho_{2}$ is the radius vector of the point of the periodic curve, $\mathrm{mm}$; $\mathrm{A}$ is the amplitude of the curve, $\mathrm{mm}$; $\mathrm{R}$ is the radius of the middle circle passing between the peaks and troughs of the curve, $\mathrm{mm} ; \mathrm{z}_{2}$ is the number of periods of the curve; $\varphi$ is the polar angle, rad.

The following equations can be used to determine kinematic characteristics of the planetary transmission with the raceway which is a circle with a center offset by the value A.

The equation of the frame velocity of a satellite ball: 


$$
v_{n}=\omega_{3}\left(\sqrt{R^{2}-A^{2} \sin ^{2} z_{2} \varphi}+A \cos z_{2} \varphi\right) .
$$

The formula for the relative velocity of the satellite ball can be obtained by differentiating the radius vector $\rho_{2}$ with respect to time $t$ and substituting the expression (1), and taking into account that the radius vector $\rho_{2}$ is a function of the relative rotation angle $\varphi_{0}=\left(\omega_{1}+\omega_{3}\right)$ t:

$$
v_{o}=A\left(\omega_{1}+\omega_{3}\right) z_{2} \sin z_{2} \varphi\left(\frac{\cos z_{2} \varphi}{\sqrt{R^{2}-A^{2} \sin ^{2} z_{2} \varphi}}+1\right),
$$

where $\omega_{1}$ is the angular velocity of the input shaft; $\omega_{3}$ is the angular velocity of the output shaft.

The frame acceleration of the satellite ball can be obtained by differentiating the expression (2) with respect to time and assuming that the satellites move at a constant angular velocity:

$$
a_{n}=\omega_{3}^{2}\left(\sqrt{R^{2}-A^{2} \sin ^{2} z_{2} \varphi}+A \cos z_{2} \varphi\right) .
$$

By differentiating the expression (3) with respect to time, we can determine the relative acceleration of the satellite ball using the formula:

$$
\begin{aligned}
& a_{0}=\frac{A^{2} z_{2}^{2}\left(\omega_{1}+\omega_{3}\right)^{2}}{\sqrt{R^{2}-A^{2} \sin ^{2} z_{2} \varphi}}\left(\frac{A^{2} \cos ^{2} z_{2} \varphi \sin ^{2} z_{2} \varphi}{R^{2}-A^{2} \sin ^{2} z_{2} \varphi}-\cos 2 z_{2} \varphi\right) \\
& -A z_{2}^{2}\left(\omega_{1}+\omega_{3}\right)^{2} \cos z_{2} \varphi .
\end{aligned}
$$

Taking into account that $\vec{\omega} \perp \vec{v}$, the Coriolis acceleration is determined from the formula:

$$
a_{k}=2 A \omega_{3}\left(\omega_{1}+\omega_{3}\right) z_{2} \sin z_{2} \varphi\left(\frac{\cos z_{2} \varphi}{\sqrt{R^{2}-A^{2} \sin ^{2} z_{2} \varphi}}+1\right)
$$

The absolute velocity of complex motion of the satellite ball is determined by the method of projections onto the coordinate axes of the coordinate system of a fixed element. Whereupon the position of the satellite ball center relative to the fixed reference system is determined by the radius vector:

$$
\vec{r}=\vec{r}_{o}+x \vec{i}+y \vec{j}+z \vec{k}
$$

where $\vec{r}_{o}$ is the radius vector of the origin of the moving relative coordinate system; $\mathrm{x}, \mathrm{y}, \mathrm{z}$ are relative coordinates; $\mathrm{i}, \mathrm{j}$, $\mathrm{k}$ are unit vectors.

The absolute velocity of the ball center is equal to the derivative of the radius vector $\vec{r}$ with respect to time $\mathrm{t}$ :

$$
\vec{v}=\frac{d \vec{r}}{d t} .
$$

Differentiating equation (8) with respect to time t, we obtain the formula of absolute velocity in vector form:

$$
\vec{v}=\frac{d \vec{r}_{o}}{d t}+x \frac{d \vec{i}}{d t}+y \frac{d \vec{j}}{d t}+z \frac{d \vec{k}}{d t}+\dot{x} \vec{i}+\dot{y j}+\dot{z} \vec{k}
$$

It is seen from the formulas obtained that the velocities and accelerations of the satellites vary periodically from zero to the maximum value. It is important that the increase and decrease in velocity and acceleration be slow and smooth. The relationship between the geometric parameters $A, \rho_{2}, R, z_{2}$ of the formula (1) for the periodic raceway is also important for increasing smoothness of transmission operation and its efficiency. The optimization of these parameters was investigated by M.F. Pashkevich [1, 2].

To assess the effect of the geometry of the transmission raceway profile on its kinematics and dynamics, the computer solid-state 3D models of transmission rotation with a preset angular velocity on the input shaft and an applied moment on the output shaft were developed in the SolidWorks Motion module. The first model is intended for a geometric profile of the periodic raceway in the form of arcs of circles of a preset radius corresponding to the radius of the satellite balls. The second model is for a geometric profile of the periodic raceway of the equidistant curve described by the equation (1). The developed computer models take into account backlashes between the balls and raceways, impacts of the satellite balls when the engagement parts rotate under load, inertia forces, and elastic deformations. This simulation of the transmission operation replaces the full-scale experiment.

Figure 1 shows the window of the SolidWorks Motion program with the formation of the periodic raceway profile from the equation (1).

Figure 2 shows a computer CAD model that was used to simulate kinematics and dynamics of the planetary radial plunger transmission. In The SolidWorks Motion program, the following simulation parameters were set for the computer dynamic model: engine speed - $100 \mathrm{rpm}$; the moment on the axis of the transmission output shaft $-20 \mathrm{Nm}$; the period for one revolution of the output shaft -8.4 seconds. The contact conditions between the satellites and raceways were also set. The gear ratio of the mechanism is 14 .

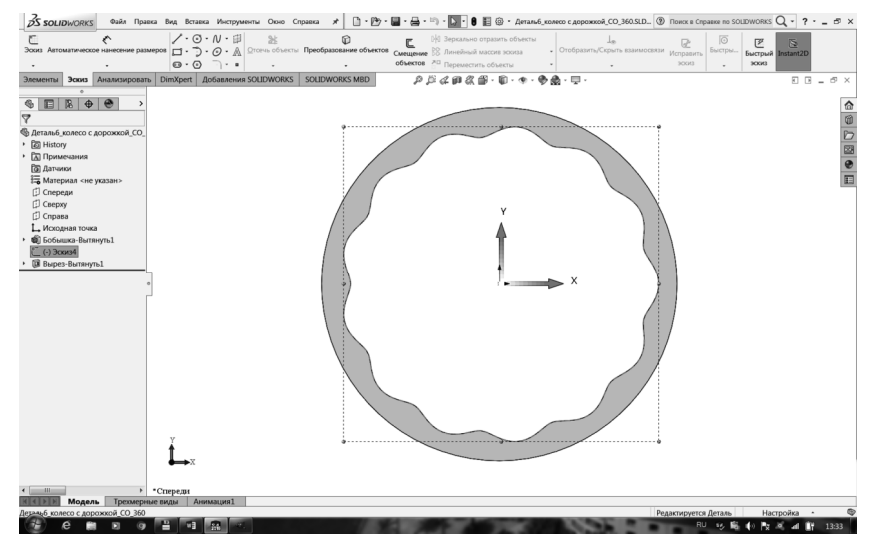

Fig. 1. The window of the SolidWorks Motion program which shows the formation of the periodic raceway profile 


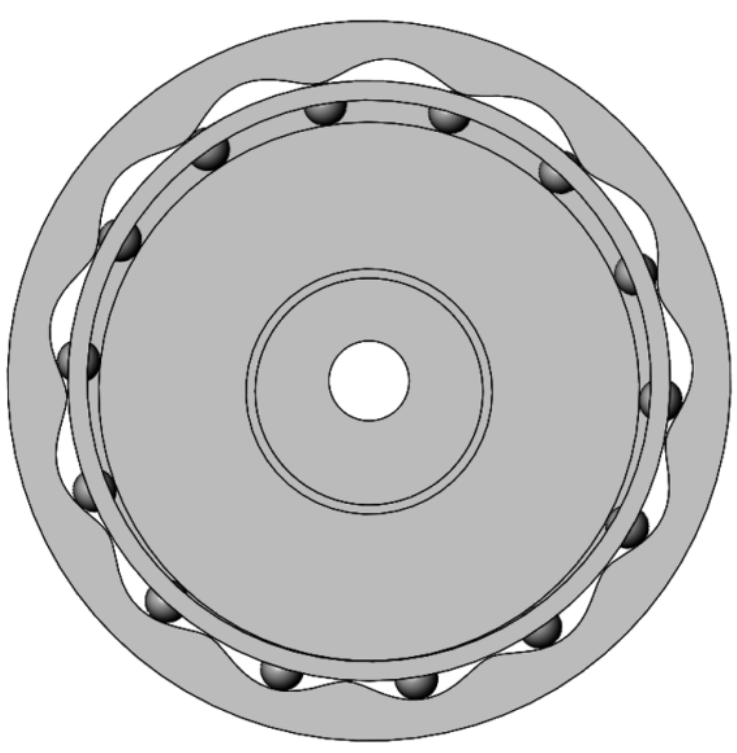

Fig. 2. Computer CAD model of the planetary transmission for simulating its kinematics and dynamics

As a result of modeling the rotation of planetary radial plunger transmission with the preset parameters given above, the diagrams and amplitude-frequency spectra of angular displacements, velocities and accelerations were obtained for the two profiles of periodic raceways. In accordance with the technique proposed by M.F. Pashkevich [13], the kinematic transmission error was calculated for different angles of rotation of its shafts. Also, diagrams and amplitude-frequency spectrum of the kinematic error were plotted. Fig. 3 shows the diagram of the kinematic transmission error $F_{k}$ with respect to the time $t$ in one complete revolution of the output shaft with the periodic raceway profile in the form of arcs of circles. Fig. 5 shows the diagram of the kinematic transmission error with respect to time during one complete revolution of the output shaft with the periodic raceway geometry constructed from the formula (1). Fig. 4 and 6 show diagrams of the amplitudefrequency spectra obtained by decomposing the values of the kinematic transmission error (Fig. 3 and 5) into a Fourier series. The diagrams (Fig. 3 and 5) show the kinematic error $F_{k}$ in degrees, and the time $t$ in seconds. The diagrams (Fig. 4 and 6) show the magnitudes of spectrum amplitudes A, n being the harmonic numbers.

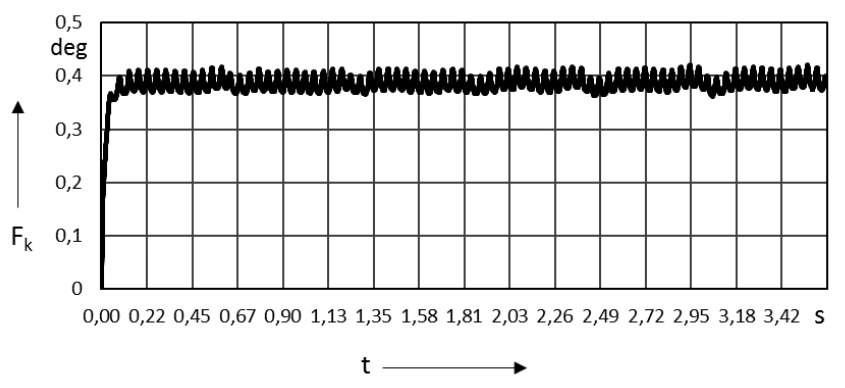

Fig. 3. The diagram of kinematic error of the planetary transmission with the periodic raceway profile in the form of arcs of circles

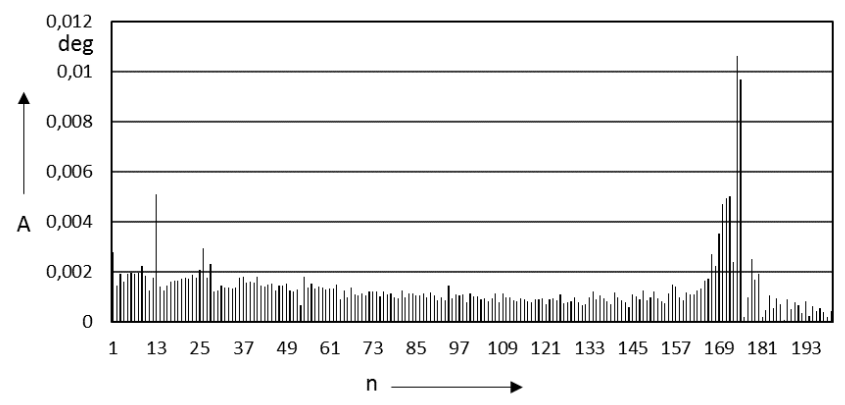

Fig. 4. The amplitude-frequency spectrum of kinematic error of the planetary transmission with the periodic raceway profile in the form of arcs of circles

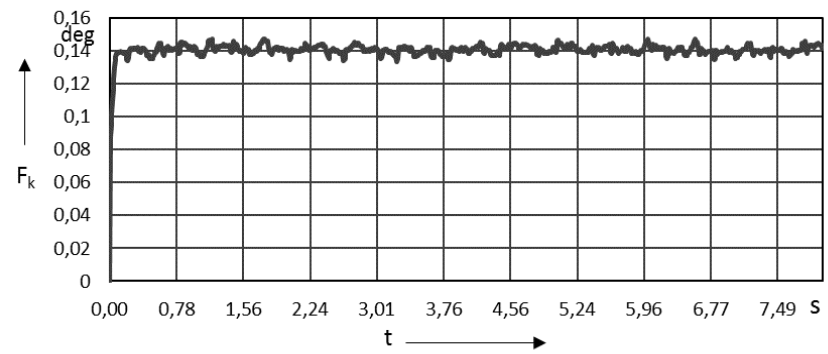

Fig. 5. The diagram of kinematic error of the planetary transmission with the periodic raceway profile constructed from the equation of an offset circle

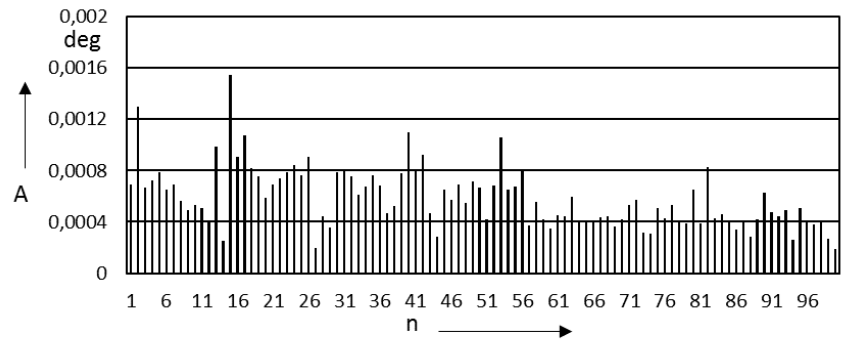

Fig. 6. The amplitude-frequency spectrum of kinematic error of the planetary transmission with the periodic raceway profile constructed from the equation of an offset circle

The diagrams (Fig. 3 - 6) show the planetary transmission with the profile of the periodic raceway, along which the satellite balls move during the rotation, formed by the equation of an offset circle (1). This transmission is characterized by significantly better kinematic characteristics (smaller amplitude and fluctuation frequency) than the transmission with a simplified form of the raceway in the form of arcs of circles.

As a result of the kinematic and dynamic simulation of the planetary radial plunger transmission with a gear ratio of 14 and two different profiles of the periodic raceways, it was established that the magnitudes of angular velocity fluctuation amplitude and kinematic error depend on dynamic loads, the imbalance of the input member, backlashes in the engagement, and impacts of satellite balls during the simulation of rotation with an applied moment on the output shaft.

Prior to and after the stabilization of rotation speed, the raceway with the profile in the form of arcs of circles had the biggest kinematic error of 0.42 and 0.057 angular minutes (the 
difference between the largest and smallest values in the diagrams, Fig. 3 and 5), respectively, and for the raceway with the profile of the curve constructed based on the equation of an offset circle these magnitudes were 0.14 and 0.013 angular minutes, respectively. When simulating the transmission rotation in the Motion module of the SolidWorks program within 0.1 second, backlashes in engagement are closed, and the kinematic error changes from zero to 0.42 degrees (Fig. 3) and from zero to 0.14 degrees (Fig.5). The biggest kinematic transmission error of the periodic raceway profile constructed based on the equation of an offset circle was three times less than the kinematic transmission error of the raceway profile in the form of arcs of circles.

Table I shows the kinematic characteristics of the planetary transmission under study with two types of profiles of the periodic raceways. The values were obtained after modeling in SolidWorks Motion and mathematical processing. Profile 1 is formed by arcs of circles of a preset radius. Profile 2 is formed by a periodic curve described by the equation of an offset circle (1).

TABLE I VALUES OF KINEMATIC CHARACTERISTICS OF THE PLANETARY RADIAL PLUNGER TRANSMISSION WITH TWO FORMS OF PERIODIC RACEWAY PROFILES

\begin{tabular}{|c|c|c|c|}
\hline $\begin{array}{c}\text { Profile type } \\
\text { of the } \\
\text { periodic } \\
\text { raceway }\end{array}$ & $\begin{array}{c}\text { Fluctuatio } \\
\text { n of } \\
\text { angular } \\
\text { velocity of } \\
\text { the output } \\
\text { shaft, } \\
\text { rpm }\end{array}$ & $\begin{array}{c}\text { Angular } \\
\text { acceleration of the } \\
\text { output shaft } \\
\text { (average value), } \\
\text { rad/s } \mathbf{s}^{2}\end{array}$ & $\begin{array}{c}\text { The largest } \\
\text { kinematic } \\
\text { error, deg }\end{array}$ \\
\hline Profile 1 & 1.24 & 4.71 & 0.42 \\
\hline Profile 2 & 0.18 & 1.60 & 0.14 \\
\hline
\end{tabular}

\section{CONCLUSIONS}

The computer model is proposed for constructing the geometric profile of the periodic raceway for the planetary radial plunger transmission based on the equation of a periodic curve in the form of a circle with an offset center. The transmission with the proposed profile is characterized by increased kinematic accuracy and smoothness of operation.

The diagrams were constructed, and the comparative analysis of kinematic errors of the planetary radial plunger transmission was performed for the two profiles of raceways of different geometrical shape. Kinematic characteristics of the transmission with the periodic raceway profile in the form of an eccentrically offset circle were improved.

It is established that the use of the planetary radial plunger transmission with the periodic raceway profile constructed from the equation of a periodic curve in the form of a circle with an offset center shows a three-fold and more increase of kinematic accuracy and smoothness of operation compared to a geometric raceway profile in the form of arcs of circles.

\section{References}

[1] M.F Pashkevich., V.V. Gerashchenko, Planetarnye sharikovye $i$ rolikovye reduktory $i$ ikh ispytaniya [Planetary ball and roller reducers and their testing], Minsk: BelNIINTI Publ., 1992.

[2] M.F. Pashkevich, V.M. Pashkevich, A.M. Pashkevich, S.V. Chertkov, Planetarnye kulachkovo-plunzhernye peredachi. Proektirovanie, kontrol' $i$ diagnostika [Planetary cam-and-plunger transmissions. Design, control and diagnostics], Mogilev: Belarusian-Russian University Publ., 2003.

[3] R.M. Ignatishchev, "Sine ball speed reducer", Mechanics of Machines, vol. 58, pp. 95-98, 1981 (in Russ.).

[4] R.M. Ignatishchev, Sinusosharikovyy reduktor [Sine ball speed reducer], Minsk: Vysheyshaya shkola Publ., 1983.

[5] M.E. Lustenkov, Peredachi s promezhutochnymi telami kacheniya: opredelenie i minimizatsiya poter' moshchnosti tipa [Transmissions with intermediate rolling elements: determination and minimization of power losses], Mogilev: Belarusian-Russian University Publ., 2010.

[6] LiXin Xu, YuHu Yang, "Dynamic modeling and contact analysis of a cycloid-pin gear mechanism with a turning arm cylindrical roller bearing", Mechanism and Machine Theory, vol. 104, pp. 327-349, October 2016

[7] Jian Wang, Shanming Luo, Deyu Su, "Multi-objective optimal design of cycloid speed reducer based on genetic algorithm", Mechanism and Machine Theory, vol. 102, pp. 135-148, August 2016.

[8] XiaoXiao Sun, Liang Han, Kaiwei Ma, Luyang Li, Jian Wang, "Lost motion analysis of CBR reducer", Mechanism and Machine Theory, vol. 120, pp. 89-106, February 2018

[9] Ta-Shi Lai, "Design and machining of the epicycloid planet gear of cycloid drives", The International Journal of Advanced Manufacturing Technology, vol. 28, issue 7-8, pp. 665-670, April 2006.

[10] Ken-Shin Lin, Kuei-Yuan Chan, Jyh-Jone Lee, "Kinematic Error Analysis and Tolerance Allocation of Cycloidal Gear Reducers", Mechanism and Machine Theory, vol. 124, pp. 73-91, June 2018.

[11] Yunhong Meng, Changlin Wu, Liping Ling, "Mathematical modeling of the transmission performance of $2 \mathrm{~K}-\mathrm{H}$ pin cycloid planetary mechanism", Mechanism and Machine Theory, vol. 42, issue 7, pp. 776790, July 2007.

[12] Linshan Han, Fei Guo, "Global sensitivity analysis of transmission accuracy for RV-type cycloid-pin drive", Journal of Mechanical Science and Technology, vol. 30, issue 3, pp. 1225-1231, March 2016.

[13] M.F. Pashkevich et al., "Controlling the accuracy of planetary roller transmissions based on their simulation and the spectral analysis of kinematic errors", Izvestiya Belorusskoy Akademii Nauk, vol. 1(13), pp. 45-52, 2002. (in Russ.).

[14] A.V. Kapitonov, K.V. Saskovets, A.I. Kasyanov, "Computer 3d modeling of designs and kinematic parameters of planetary smalldimensioned transmissions", Vestnik Polotskogo gosudarstvennogo universiteta, vol. 11, pp. 34-40, 2010 (in Russ.).

[15] A.V. Kapitonov, S.G. Chernyakov, K.V. Saskovets, A.I. Kasyanov, "Methods of experimental research into kinematic precision of planetary transmissions with intermediate rolling bodies and monitoring the profile of raceways". Vestnik Belorussko-Rossiyskogo universiteta, vol. 2 (51), pp. 41-50, 2016 (in Russ.). 